\title{
Cysticercosis in cattle and its public health implications in Mekelle City and surrounding areas, Ethiopia
}

Getachew Abay ${ }^{l}$ and Ashwani Kumar ${ }^{l}$ *

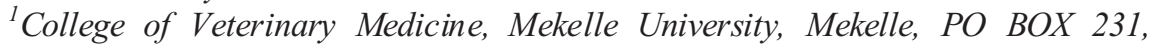
Ethiopia

*Corresponding author: ashwanivet126@yahoo.com

\begin{abstract}
To detect bovine cysticercosis, postmortem examination was done on 1023 cattle coming to Mekelle abattoir from highland and lowland areas of Mekelle and its surroundings. Routine examination of masseter, triceps and diaphragmatic muscles, heart, liver, lung and the tongue was made. True viability of the cyst was ascertained by keeping the cysts in 5\% sodium taurocholate overnight and evagination of scolex indicated cyst's viability while the dead ones remained intact. Data from Governmental and nongovernmental health centers was collected to find the cases of human taeniaisis in the study areas. Seventy four cattle were positive for cysticercosis giving a percent positivity of $7.23 \%$. Cysticercus bovis were found at different sites, viz. masseter muscles, triceps muscles, heart, liver, tongue and lungs, but the livers of 69(6.74\%) of 1023 cattle were found positive for one or more cysts and this positivity was higher than that of the other sites. Out of the cysts from 10 positive carcasses tested for viability, seven animals were having viable infection. Analysis of data from Governmental and non-governmental health centers on7171human patients' positive for various parasitic infections revealed Taenia saginata in 23. Presence of taenaisis/cysticercosis in man and animals highlights the public health significance of this infection.
\end{abstract}

Key words: Cysticercosis, Cysticercus bovis, occurrence, taeniasis, Taenia saginata,

http://dx.doi.org/10.4314/evj.v17i1.3

\section{Introduction}

Bovine cysticercosis is the infection of the cattle caused by Cysticercus bovis and consumption of the raw or undercooked meat results in human taeniasis. Also, the infection in cattle has economic significance due to the total or partial 
condemnation of carcasses or their reduced value due to refrigeration, extra handling, and transport. Live cattle having $C$. bovis show no symptoms and the presence of the cysts in carcasses is observed visually during the routine meat inspection by making different incisions into muscles and organs. Human taeniasis is common in Ethiopia, as the practice of eating raw or undercooked beef is not uncommon in many sections of human population (Feseha Gebreab, 1995). Commonly used herbal drugs as taenicides are unable to eliminate the parasite and thus proglottids are passed out with faecal matter and become the source of infection for cattle (Shibru Tedla, 1986). T. saginata/C.bovis has been recorded from different regions and charted cities of Ethiopia, viz, at Addis Ababa abattoir Nigatu Kebede et al., (2009), in Southern Ethiopia Alemayehu Regassa et al. (2009), Northern Ethiopia Getachew Abay, (2008); Kumar and Gebretsadik Berhe, (2008), Awassa municipal abattoir (Southern Nations' Nationalities' Peoples' Region) Fufa Abunna et al., (2008), Amhara National Regional State, northwestern Ethiopia Nigatu Kebede, (2008), Bahir Dar (Amhara region) Mulugeta Alemu, (1997), north Gonder zone of Ethiopia Hailu Degefu, (2005) and Hailu Degefu (2005) in selected areas of eastern Shoa. As compared to report of low prevalence in northern part of the country Kumar and Gebretsadik Berhe, (2008) and Getachew Abay, (2008), bovine cysticercosis is prevalent at a higher rate from southern, western and northwestern parts of the country (Fufa Abunna et al., 2008, Nigatu Kebede, 2008, Ahemad Ibrahim, 1990). Keeping in view the public health significance of bovine cysticercosis, the present study was proposed to find the occurrence of bovine cysticercosis in some parts of Northern Ethiopia and its implications on public health.

\section{Materials and Methods}

\section{Study area}

The present study was conducted from November 23, 2007 to February 27, 2008 at Mekelle Municipality Abattoir. Mekelle is the capital city of Tigray Regional State and is located $783 \mathrm{kms}$ North of Addis Ababa. The total area of the city is estimated to be $53 \mathrm{~km}^{2}$ and it is located between altitudes of 2000-2200 m above sea level and has moderate (Woynadega) zone of climatic conditions. Geographically, Mekelle city is found in $39^{\circ} 28^{\prime}$ east and $13^{\circ} 32^{\prime}$ north. The average annual rainfall of the city ranges from $50-250 \mathrm{~mm}$ and the average mean temperature is $19^{\circ} \mathrm{C}$. The total population of the city is around 200,000 comprising 97,200 (48.6\%) male and $102,800(51.4 \%)$ female. 
Ante-mortem and postmortem examination

The study animals comprise indigenous cattle slaughtered at Mekelle agricultural abattoir. The cattle slaughtered in this abattoir come from different sites (areas) of the region. The total population of cattle in the region is estimated to be 3.37 million (BoANR, 1999). The animals were examined before slaughter (ante mortem inspection) and after slaughter (postmortem inspection). During antemortem examination each animal was marked for identification by writing a code on its head by using unwashable ink.

During postmortem inspection, examination of muscles and different organs for the presence of cysts was undertaken by giving incisions. The various sites examined were masseter muscle, heart, liver, lung, triceps muscle, diaphragmatic muscle and the tongue. The presence of infection of $C$. bovis at a site was categorized as low, moderate or heavy depending on the number of cysts and basis of such classification was Meat Inspection judgment described by Kenyan Meat Control Act, 1977. If the number of cysts at a site were less than 10, it was taken as low, 10 to less than 20 as moderate and greater than 20 as heavy infection.

\section{Cyst viability test}

The viability of the cysts was tested by putting them in 5\% sodium taurocholate in saline solution, and incubated at $37^{\circ} \mathrm{C}$ for $1-2$ hours in the incubator. Evagination of the scolex in viable cysts normally takes place within 1-2hrs Gracey et al., (1999) but were examined till 24 hours of incubation (Wanzala et al., 2003). Due to unavailability or limitation of chemical (sodium taurocholate) in the earlier period of this study, the viability test was could be done on cysts from 10 of 74 positive carcasses.

\section{Taeniosis}

Efforts were made to collect data from Mekelle city on human beings carrying $T$. saginata infection. For this, four Governmental and one Nongovernmental health centers were visited from September, 2007 -April, 2008.

Data analysis

The occurrence of $C$. bovis related to specific factors was determined as the proportion of affected cattle out of the total examined. 


\section{Results}

From December 2007 to February 2008, 1023 cattle were examined both at the time of antemortem and postmortem examination. Out of the total 1023 cattle, 503 were from highland while 520 cattle originated from lowland areas. Age wise, these animals were divided into 366 as adult animals between the age groups of 2-5 years and 657 as old cattle of more than 5 years of age. Out of the total 1023 cattle examined, 74 were found positive for the presence of $C$. bovis giving a percent positivity of $7.23 \%$.

Regarding cyst locations, out of the total of 74 cattle positive for $C$. bovis, carcasses of 70 cattle were having the cysts at one site only and 4 at more than one site (Table-1). C. bovis were found in different organs; however its presence was maximum in livers. Out of total 1023 cattle examined, livers of 69 (6.74\%) animals were having these cysts (Fig.1). The other organs of cattle carcass found positive for $C$. bovis were tongue, $4(0.39 \%)$, triceps muscles, $3(0.29 \%)$, heart, $4(0.39 \%)$, masseter muscles, $1(0.097 \%)$ and lung 3(0.29\%). Depending upon the number of cysts, the infection of cysticercus was recorded as high in 33(44.59\%), moderate in $18(24.32)$ and low in $23(31.08 \%)$ cattle. It was moderate to high in livers while low in other sites. Out of the cysts from 10 positive carcasess tested for viability; 7 animals were having viable infection. T. saginata in human population of Mekelle city: Out of the 7171patients visiting the 5 health centers in Mekelle and positive for various parasitic infections, 23 were reportd to be positive for T. saginata infection.

Table 1: Number of carcasses having Cysticercus bovis at different sites

\begin{tabular}{|c|c|c|c|}
\hline \multicolumn{2}{|c|}{ Cysts present at one site } & \multicolumn{2}{|c|}{ Cysts present at more than one site } \\
\hline Site & Number of carcasses & Sites & Number of carcasses \\
\hline Masseter muscles & 01 & Liver + Heart & 01 \\
\hline Triceps muscles & 01 & Liver+ lung +triceps & 01 \\
\hline Tongue & 02 & Liver + heart + tongue + lung & 01 \\
\hline Heart & 01 & & \\
\hline Liver & 61 & Liver+heart+tongue + Lung+triceps & 01 \\
\hline
\end{tabular}




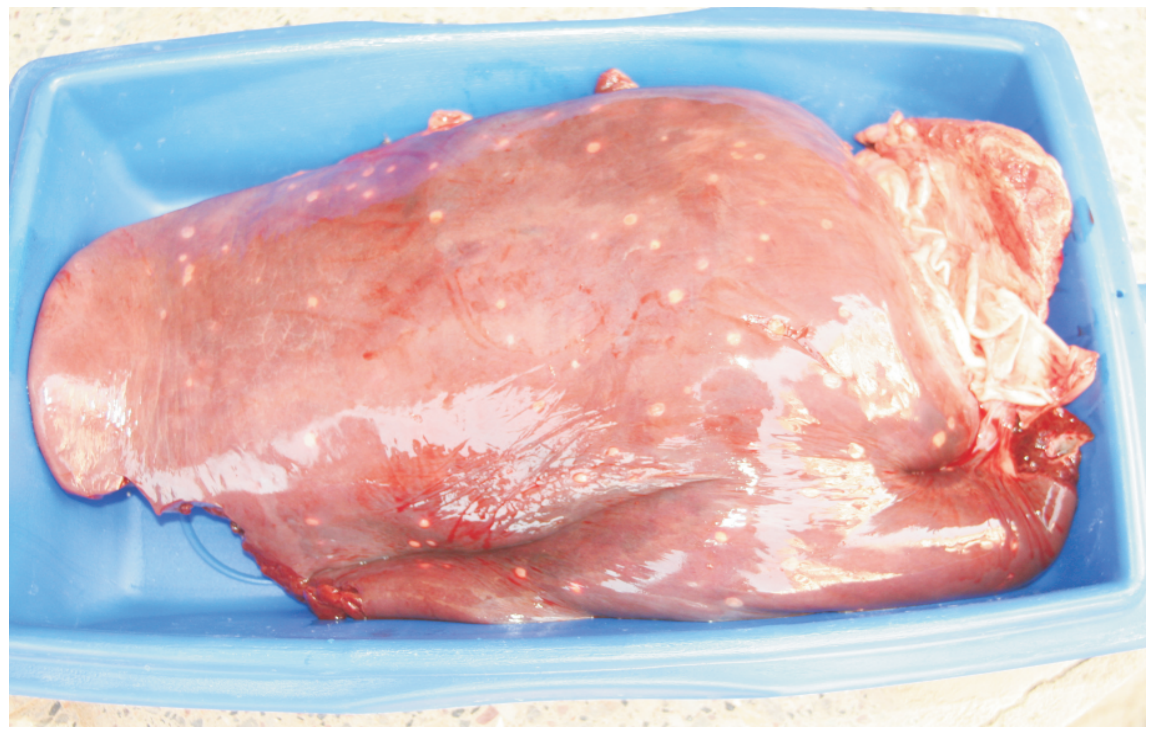

Figure 1. Bovine liver showing cysts of Cysticercus

\section{Discussion}

The prevalence of $C$. bovis is low in developed countries, being less than $1 \%$ of carcasses inspected (Onyango - Abuje et al., 1996). However, the infection rate is often around 30\% in developing countries (Maxwell et al., 2006). In the present study, the occurrence rate of cysticercosis in Mekelle city was found as 7.23\% which is similar to $8.28 \%$ reported earlier from the same area by Kumar and Gebretsadik Berhe, (2007). However, the percent positivity of $7.23 \%$ is lower than the reported from other developing countries. This may be due to the fact that the incidence of C. bovis varies from country to country and also reflects the expertise of meat inspectors. Moreover, in the routine inspection of beef carcasses, there is practical limitation to the number and degree of incisions permissible, for gross mutilation lowers the marketability of the carcass, as a result many infestations remain undetected. The flooding of pastures, use of sewage effluent for pasture irrigation without adequate treatment, free access of cattle to surface water, proximity of wastewater effluent, keeping the animals indoor, farm sanitation, farmer's education about the disease are the other factors that make the difference in prevalence percent of bovine cysticercosis. In Ethiopia, available literature 
reveals different percentages of cattle positive for $C$. bovis. Tembo (2001) found $70(3.11 \%)$ of 2250 randomly selected adult of bovine carcasses in central highlands (Akaki, Debrezeit, Nazereth) positive for cysticercosis. Similarly, in Gondar area, the prevalence of $C$. bovis was reported to be $9.7 \%$ and $4.9 \%$ by Amsalu Demissie, (1989) and Shimelis Dawit (2004) respectively. In contrast to low prevalence reported above, such studies in export abattoirs like Mojo, ELFORA, Dukem, Luna, revealed a comparatively higher prevalence of cysticercosis in cattle. The percent positivity in these abattoirs was $17.9 \%, 13.6 \%$, 19.2\%, and 27.6\% respectively (Hailu Degefu, 2005). In Amhara National regional state, Nigatu Kebede (2008) in his study from September, 2005 to February, 2007, showed the presence of $C$. bovis in $824(18.49 \%)$ of 4456 cattle. From these reports, it is evident that there is variation in the prevalence of $C$. bovis in cattle within Ethiopia. Gracey et al., (1999) viewed that difference in the prevalence of cysticercosis within a country probably reflects the difference in the expertise/or diligence of meat inspectors. In addition to this, the incidence or prevalence of cysticercosis is reported high in modern and export abattoirs which may be due to a thorough meat inspection by incising all organs/muscles and also inspectors in such abattoirs are more experienced and also have awareness to the economic importance of $C$. bovis in the exportation.

C. bovis was recorded at various sites on the infected carcasses. They were present in liver of $6.74 \%$, hearts of $0.39 \%$, tongues of $0.29 \%$, triceps muscles of $0.29 \%$, lungs of $0.29 \%$ and masseter muscles of $0.097 \%$ cattle. C. bovis are commonly found in muscles of mastication, particularly masseter muscles, shoulder muscles, heart, tongue, diaphragm and occasionally in fat, liver, lungs and lymph nodes. In the present study cysts were found in liver, heart, tongue, triceps, lungs and masseter muscles with predominance in the liver showing a positivity of $6.74 \%$ of 1023 cattle. According to Wanzala et al., (2003), all the parts of carcasses were equally important as predilection sites for cysticerci and could be equally used during routine meat inspection at slaughterhouses except for rumen, fat layers, spleen and skin. They reported $22 \%$ of bovine livers positive for cysticerci. From several reports, variation and deviation in localization of cysts are quite possible. For example, in a study at Addis Ababa abattoir, the infection rate of $C$. bovis was as high as $16.3 \%$ in fore legs (Feseha Gebreab, 1995). C. bovis has also been reported from 3.7\% cattle livers and 1.6\% in lungs (Fiseha Gebreab, 1995). Similarly in east Shoa Oromia regional state, $4.74 \%$ carcasses examined were positive for this infection in liver and $0.7 \%$ in lungs (Hailu Degefu, 2005). Another reason of reporting a higher percentage of livers' positivity for $C$. bovis infection is imperfect meat inspection facilities at abattoir in Mekelle. In this abattoir, the 
organs and muscles incised for inspection were liver, heart, lungs, tongue masseter muscle and triceps muscles. Even in these sites the number of incision made was limited to one in most of the cases. The other heavy muscles, like fore legs, neck muscle, hind legs and shoulder muscled were usually not inspected. This is because the butchers and owners usually oppose several incisions due to loss of earnings. As compared to inspection of predilection sites of C. bovis, thorough examination of livers were observed to be the practice at Mekelle abattoir and this may be another reason of reporting positive livers in higher numbers in this study than the other sites including the striated muscle "as the predilection site.

From the data of this survey, it is evident that 70 of 74 positive animals, the cysts were found only at one site; while one each of 74 carcasses was having cysts at two, three, four and five locations. The data citing the number of sites in a carcass showing cysts of $C$. bovis infected carcasses is scanty. Kumar and Gebretsadik Berhe (2007) reported the presence of cysts in one to three locations in a carcass.

Bovine cysticercosis has its own public health significance. The cysts after ingestion by human beings develop into adult parasite, $T$. saginata, leading to anorexia, loss of weight, abdominal pain, digestive disturbances and insomnia. Sometimes the mobile gravid segments of adult parasite may make their way to unusual sites such as the appendix, uterus or biliary tract and may cause serious disorders. Based on the limited data collected from four governmental and nongovernmental health centers in Mekelle, T. saginata infection was found in patients visited for treatment. To correlate exactly bovine cysticercosis with human taeniosis, further studies should be taken by collecting detailed data from all the governmental and private hospitals of the area.

Cysticercosis in cattle carcasses are of economic significance because heavily infested carcasses/organs are to be condemned while cysts in low number are to be made inactive before releasing for human consumption. As evident from the results of this study, livers of several cattle should be condemned on account of $C$. bovis infection, and remaining organs and muscles should be put to cold or hot conditioning which further enhances the cost of meat production.

\section{Conclusions}

From the present study, it can be concluded that bovine cysticercosis was prevalent in Mekelle and its adjoining areas and its percent prevalence was comparable to other parts of Ethiopia. However, the prevalence rate was lower than the ones 
reported by export abattoirs in this country. Based on cysts viability results, the risk of contracting taeniois in Mekelle would be high as most of the cysts in many carcasses tested in this study were viable and possibly may be infective coupled with the raw meat eating habit of the community. The study further revealed site (liver) on carcasses other than predilection ones having cysts in higher percent and therefore all the sites should be thoroughly examined for the cysts. A controlled study is needed to rule out the effects of factors, like livestock husbandry practices, tapeworm carrier on the farm, poor hygiene of farm staff (e.g. handling of bovine feed, or hand milking with egg-contaminated hands),defecation by man in places frequented by cattle, or in cattle feed production areas, application of human sewage slurry on pastures or highly permeable soils with possible contamination of water-bearing surface and drinking water, eggs in effluent water from sewage treatment plants, on the prevalence of C.bovis infection in cattle.

\section{References}

Abunna, F., Tilahun, G., Megersa, B., Regassa, A., and Kumsa, B., 2008. Bovine cysticercosis in cattle slaughtered at Awassa municipal abattoir, Ethiopia. Zoon. Publ. Hlth. 55(2), 82-88

Alemu, M., 1997. Bovine Cysticercosis Prevalence Economic and Public Health Importance at Bahir Dar Municipality Abattoir. (Unpublished DVM thesis, Faculty of Veterinary Medicine, Addis Ababa University, Ethiopia)

BoNAR 1999. Livestock Census. Regional State of Tigray, Bureau of Agriculture and Natural Resources. Mekelle, Ethiopia. Vol. 1. Daltons supplies Ltd Cattle and Pig weighing Tape.

Dawit, S., 2004. Epidemiology of Taenia saginata Taeniasis and cysticercosis in north Gondar Zone, north western Ethiopia .DVM thesis, Addis Ababa University, Ethiopia.

Degefu, H., 2005. Prevalence and risk factors for Taenia saginata Taeniasis/cysticercosis in three selected areas of eastern Shoa. MSc thesis, Addis Ababa University, Debrezeit, Ethiopia.

Demissie, A., 1989. Prevalence and significance Cysticercus bovis among cattle slaughter at Gondar meat factory. DVM thesis, Addis Ababa University, Debrezeit, Ethiopia. 
Gebreab, F., 1995. Zoonotic Disease in Ethiopia. Ethiopian Society of Animal production (ESAP), Addis Ababa. pp, 32-37.

Abay, G., A., 2008. A study on the prevalence and public health importance of Cysticercus bovis in Mekelle abattoir (Unpublished DVM thesis, Mekelle University, Mekelle, Ethiopia).

Gracey, J., Collins, D.S. and Huey, R., 1999. Diseases caused by helminthes and arthropod parasites. In: Meat Hygiene. 10th edition, W.B.Saunders Company Ltd., London, pp673.

Ibrahim, A., 1990. Bovine cysticercosis in animals slaughtered in Nekemte Municipality slaughter house (Unpublished DVM thesis, Addis Ababa University, Debrezeit, Ethiopia).

Kebede, N., 2008. Cysticercosis of slaughtered cattle in northwestern Ethiopia. Res.Vet. Sci., 85, 522526.

Kebede, N., Tilahun, G. and Hailu, A. (2009). Current status of bovine cysticercosis of slaughtered cattle in Addis Ababa Abattoir, Ethiopia. Trop. Anim. Hlth and Prod. 41(3), 291-4.

Kenya Meat Control Act. Kenya Government Printers; 1977. (Cited by Wanzala, 2003).

Kumar, Ashwani and Gebretsadik, B., 2008. Occurrence of cysticercosis in cattle of parts of Tigray region of Ethiopia. Haryana Vet., 47, 88-90.

Maxwell, O.N., Ukpong, U.M., Okoli, I.C. and Anosike, J.C., 2006. Cysticercosis of slaughtered cattle in Southern Nigeria. Annals of the New York Academy of Sciences. 1081, 339-346.

Onyango- Abuje, J.A., Hughes, G.O.M., Niginyi, K.M., Rugutt, M.K., Wright, S.H. and Harrison, L.J., 1996. Diagnosis of Taenia saginata cysticercosis in Kenyan cattle by antibody and antigen ELISA. Vet. Parasitogy, 61, 221-230.

Regassa ,A., Abunna, F., Mulugeta, A., Megersa, B., 2009. Major metacestodes in cattle slaughtered at Wolaita Soddo Municipal abattoir, Southern Ethiopia: prevalence, cyst viability, organ distribution and socioeconomic implications. Trop. Anim. Hlth. and Prod. 41(7), 1495-502.

Tedla, S., 1986. Introduction to Parasitology: Protozoan and Helminth parasites of man 1st edition, Addis Ababa University press, Ethiopia. 
Tembo, A., 2001. Epidemiology of T. saginata taeniasis/cysticercosis in three selected agroclimatic zones in central high land of Ethiopia, M.Sc. thesis, Addis Ababa University, Debrezeit, Ethiopia.

Wanzala, W., Onyango-Abuje, J.A., Kang'ethe, E.K., Zessin, K. H., Kyule, N.M., Baumann, M.P.O., Ochanda, H. and Harrison,L.J.S., 2003. Control of Taenia saginata by postmortem examination of carcasses. Afri. Hlth. Sci. 3(2), 68-76. 